\title{
Complexity Analysis and Control in Time-Delay Vaccine Supply Chain considering Cold Chain Transportation
}

\author{
Daoming Dai, ${ }^{1,2}$ Xuanyu Wu $\mathbb{D}^{1},{ }^{1}$ and Fengshan $S i^{1}$ \\ ${ }^{1}$ School of Management Science and Engineering, Anhui University of Finance and Economics, Bengbu 233030, China \\ ${ }^{2}$ School of Management, Hefei University of Technology, Hefei 230009, China \\ Correspondence should be addressed to Xuanyu Wu; 20142392@aufe.edu.cn
}

Received 11 May 2020; Revised 13 August 2020; Accepted 22 September 2020; Published 6 October 2020

Academic Editor: Ramachandran Raja

Copyright ( 2020 Daoming Dai et al. This is an open access article distributed under the Creative Commons Attribution License, which permits unrestricted use, distribution, and reproduction in any medium, provided the original work is properly cited.

Due to the important role of the vaccines in the prevention of global epidemics, this paper focuses on a vaccine transportation supply chain composed of one distributor and one retailer. Based on the assumption that the decision-maker does not make a decision instantaneously, we present a decision-making time-delay model. Firstly, we captured some sufficient conditions of delay-induced bifurcation for the model by regarding different combinations of the decision delay periods as the bifurcation parameters and analyzed how the speed of decision-making adjustment of distributor or retailer affects the critical point of system stability. Secondly, we made a numerical simulation on the model by using a two-dimensional bifurcation diagram, largest Lyapunov exponent, and entropy and chaotic attractor, respectively. Finally, we used two coordination methods to control chaos and compared them. The results show that when the decision delay exceeds a certain threshold, the system will lose stability or go into chaos. The precipitous speed of decision variable adjustment of the distributor or retailer will increase the entropy of the system and lead the system into a chaotic state. When the vaccine supply chain is in chaos, the effect of external control on chaos is better than that of internal control on chaos.

\section{Introduction}

Due to the important role of vaccines in the prevention of infectious diseases spread, researchers have been interested in the vaccine supply chain from many perspectives. It has been proven that vaccination is the most effective method to stop the transmission of all kinds of infectious diseases [1]. For example, regular measles vaccination and supplementary immunization activities greatly reduce the incidence rate and mortality of measles worldwide. It was estimated that measles vaccination had averted about 23.2 million deaths during 2000-2018 [2]. Unfortunately, there are still tens of millions of children who have been not covered by routine vaccines in low and middle-income countries and there are also considerable challenges in achieving a high level of vaccination coverage. One of the main obstacles to the delivery of vaccines is that strict cold chain condition requires vaccines to be stored between $2^{\circ} \mathrm{C}$ and $8^{\circ} \mathrm{C}$ at all times [3].
The transport conditions of vaccines are noticeable problems at present. Lee and Haidari [4] explored how vaccine supply chains may make substantial influence on the decision-making of ten examples of different members in the vaccine community. According to many open questions, including how the devices should be sized, the interaction between their price and size, and how often they should be replenished, Chen et al. [5] examined a model that captured the various tradeoffs in order to better guide the development of passive cold storage devices. Lemmens et al. [6] examined whether decisions at the strategic, tactical, and operational levels can figure out key issues in the vaccine supply chain, such as cold chain delivery, limited shelf life, and access to remote region. Shittu et al. [7] established a simulation model to examine the effects of variance in vaccine supply or demand on storage capacity requirements, and the results showed that the storage of vaccine can be reduced to $30 \%$ by reorganizing the supply chain. Leidner et al. [8] assessed the ability of different types of vaccine 
storage devices to maintain an appropriate temperature for vaccine storage and identify deviations from the recommended temperature. In addition, unreliable power systems and poor road conditions often lead to cold chain failures in many developing countries [9].

From operation management perspective, some researchers have explored coordination of a vaccine supply chain. Tebbens et al. [10] proposed a mathematical framework of a vaccine stockpile over time and discussed the problems of capacity constraints, production and filling delay in the development and use of the polio vaccine reserve. Brison and Letallec [11] thought that one of the common factors limiting full and fair access to effective immunization was the gap between cold chain and logistics system. Lin et al. [12] examined that the distributor decided whether a cold chain or noncold chain is used to transport vaccines, while the retailer checked when the vaccine in a vaccine supply chain is received. Dai et al. [13] presented the delivery-time-dependent quantity flexibility contract and the late-rebate contract when there were uncertainties in the design, delivery, and demand of influenza vaccine based on the background of the influenza vaccine industry. Based on the profit goal and social responsibility of foreign vaccine suppliers, Niu et al. [14] explored two typical channel structures (i.e., exclusive retailing and competitive retailing) with the overseas vaccine supplier's profit and social responsibility objectives and acquired equilibrium on vaccine prices, supply quantities, and vaccine supplier's utilities.

Many scholars have conducted chaotic behavior analysis on various aspects of supply chain. Xie and Ma [15] investigated the game between two recyclers and one processor in a duopoly market of color TV recycling and analyzed the response of the system to government decision-making. $\mathrm{Wu}$ and $\mathrm{Ma}$ [16] considered the dynamics of the game model of the epiphytic supply chain with two players and horizontal diversification of products and analyzed the equilibrium point and stable regions. Li et al. [17] established Nash game model and Stackelberg game model in multichannel supply chain and discussed the entropy diagram, the largest Lyapunov exponent and the chaotic attractor. Tu et al. [18] considered a dynamic hybrid supply chain model composed of two manufacturers and one retailer and explored the Nash equilibrium point and its stability region. Zhou and Chen [19] established two dynamic Stackelberg game models without (with) unit profit allocation and analyze the influence of relevant parameters on dynamic system stability, complexity, and channel revenue.

Some scholars have been interested in stability problems of system with time delay. Zou et al. [20] established a delayed susceptible-infectious-recovered model for the transmission of porcine reproductive respiratory syndrome virus and acquired properties of the Hopf bifurcation by the normal form theory and center manifold theorem. Zhang et al. [21] proposed a delayed tobacco smoking model containing users in the form of snuffing and presented global exponential stability results for smoking present equilibrium by LMI techniques. Rajchakit and Rajchakit [22] investigated mean square robust stability of stochastic switched discrete-time-delay systems with convex polytopic uncertainties. Li and Liu [23] examined the bullwhip effect problem of supply chain with vendor order placement lead time delays, and the results showed that vendor order placement lead time delays played an important role in supply chain dynamics and contributed to its turbulence and volatility. References [24-28] analyzed the stability of several complex neural networks with kinds of time delays by using a Lyapunov-Krasovskii. In addition, Pratap et al. [29] explored the global existence of Filippov solutions and the robust generalized Mittag-Leffler synchronization of fractional order neural networks with discontinuous activation and impulses. References $[30,31]$ are devoted to the results of dissipative control for kinds of switched neutral timedelayed systems whose sufficient conditions for boundedness are obtained by using Lyapunov stability theory. References [32, 33] discussed the local stability and Hopf bifurcation of kinds of viruses (heroin or worm) model with time-delay and investigated the properties of the Hopf bifurcation by using center manifold theorem and the normal form theory. References [34, 35] investigated the problems of robust dissipative control for kinds of discrete-time systems and analyzed a set of sufficient conditions by using Lyapunov technique and linear matrix inequality approach.

As far as we know, most existing researches on vaccine supply chains are related to medical research and public environmental health. Many scholars have been interested in vaccine supply chains from operation management perspective, but few of them are involved to stability of the channel system with time-delay. Many existing researches assume that decision-makers make decisions instantaneously, but decisions with time-delay often occur for information transmission delay. Thus, it is more practical that time-delay is introduced into a vaccine supply chain. In this paper, we consider a time-delay supply chain composed of a distributor and a retailer, in which the for-profit distributor purchases temperature-sensitive vaccines from the manufacturer and then resells them to the retailer. Because the vaccines become inactive in storage at unsuitable temperature, the distributor chooses the transportation mode with cold chain and increases the order quantity of the vaccines. The retailer often checks the effect of cold chain transportation and makes the distributor choose an appropriate cold chain transportation mode. We established a time-delay model of dynamic decision-making and analyzed the influence of time-delay parameter on the stability of supply chain system. We not only illustrated the Hopf bifurcation diagram of the system but also derived the condition that the adjustment speeds of the decision variables led to the period-doubling bifurcation of the system. In addition, we analyzed the chaotic behavior of the system by abundant simulation diagrams, such as Lyapunov exponent, entropy, and chaotic attractor.

The remainder of this paper is organized as follows. The model conceptualization and the assumptions of the problem are described in Section 2. The problem is modeled in Section 3. Equilibrium points and Hopf bifurcation are analyzed in Section 4. Numerical analysis and managerial insights are presented in Section 5. Chaos control is discussed in Section 6. Section 7 concludes this paper with discussion of the possible future research direction. 


\section{Description and Assumption of the Problem}

2.1. Model Construction and Assumptions. This paper mainly considers a vaccine supply chain composed of a distributor and a retailer in which the for-profit distributor purchases temperature-sensitive vaccines from the manufacturer and then resells them to the retailer. Because the vaccines become inactive in storage at unsuitable temperature, the distributor chooses the cold chain mode of transportation and increases the order quantity of the vaccine. The retailer often checks the effects of cold chain transportation that the distributors chose to make them choose a cold chain transportation mode that is as appropriate as possible. The structure of vaccine supply chain system is shown in Figure 1.

The main assumptions of this paper are as follows.

(i) The distributor and the retailer are bounded rational.

(ii) This paper only considers one-time investment. The distributor uses a cold chain to transport vaccines, the cold chain transportation level that needs to be invested is $y$, and distributor incurs the investment cost $k_{1} y^{2} / 2$. The retailer checks whether vaccines are transported by cold chain with the inspection level $s$ and incurs the inspection cost $k_{2} s^{2} / 2$, where $k_{1}$ and $k_{2}$ are investment parameter and inspection parameter, respectively.

(iii) In order to ensure that distributor and the retailer can make normal profits, let $p>w>c$.

(iv) In order to ensure the vaccines' activity, the distributor must choose the cold chain to transport the vaccines, and retailer will check the cold chain transportation of vaccines to ensure the vaccines' activity.

(v) The influence of $y$ on demand function is greater than the influence of $s$, so $\eta_{1}>\eta_{2}$.

2.2. Symbolic Description. The meanings of $\alpha, \beta, p, w$, and $c$ are described concisely in Table 1.

The demand $D$ is considered as a function on transportation level, inspection level, and selling price of the vaccines. The functional form of market demand on the vaccines can be written as follows [36]:

$$
D=\alpha(1-\beta p)\left(\eta_{1} y+\eta_{2} s\right)
$$

where $\eta_{1}$ and $\eta_{2}$ represent the influence coefficients of the distributor's cold chain transportation level $y$ and the retailer's inspection level $s$ on demand, respectively.

\section{Multiperiod Decision-Making Game Model with Delay}

The profit functions of the distributor and retailer are expressed as follows:

$$
\begin{aligned}
& \pi_{d}=(w-c) \alpha(1-\beta p)\left(\eta_{1} y+\eta_{2} s\right)-\frac{k_{1} y^{2}}{2}, \\
& \pi_{r}=(p-w) \alpha(1-\beta p)\left(\eta_{1} y+\eta_{2} s\right)-\frac{k_{2} s^{2}}{2} .
\end{aligned}
$$

From (2) and (3), the decision variable of the distributor is $y$, and the retailer's decision variables are $p$ and $s$. Thus, the marginal profit function can be written as

$$
\left\{\begin{array}{l}
\frac{\partial \pi_{d}}{\partial y}=\eta_{1}(w-c) \alpha(1-\beta p)-k_{1} y \\
\frac{\partial \pi_{r}}{\partial p}=\alpha(1-2 \beta p+\beta w)\left(\eta_{1} y+\eta_{2} s\right) \\
\frac{\partial \pi_{r}}{\partial s}=\eta_{2}(p-w) \alpha(1-\beta p)-k_{2} s .
\end{array}\right.
$$

Because of the complexity of the market, the distributor and retailer do not fully know the market demand and the decisions of the competitors in the most cases. Therefore, they adjust their own decisions according to their marginal profit. The distributor and retailer make decisions based on bounded rationality. The values of decision variables in period $t+1$ under bounded rational decision are the values of decision variables at period $t$ plus the changes of decision variables at period $t$.

$$
\left\{\begin{array}{l}
y(t+1)=y(t)+v_{1} y(t) \frac{\partial \pi_{d}}{\partial y} \\
p(t+1)=p(t)+v_{2} p(t) \frac{\partial \pi_{r}}{\partial p} \\
s(t+1)=s(t)+v_{3} s(t) \frac{\partial \pi_{r}}{\partial s}
\end{array}\right.
$$

where $v_{1}, v_{2}, v_{3}$ are the decision variables adjusting speed of the distributor and the retailer, respectively.

In classical research on supply chains by using dynamics, scholars always assumed that decision-makers make decisions instantaneously; however, this assumption seems not to be realistic, because decision-makers are often bounded rational and risk averse and need to be considered before making a decision. $\tau$ is the time of the decision-maker's recognition of the need to take a decision to making a decision. So, we introduce the time-delay parameter into the differential equations. 


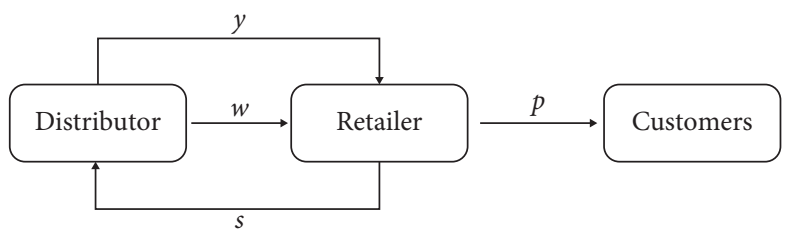

FIgURE 1: The vaccine supply chain system.

TABLE 1: The parameters description for the system.

\begin{tabular}{lc}
\hline$\alpha$ & The initial demand \\
$p$ & Retail price of unit vaccine \\
$\beta$ & Sensitivity coefficient of consumers to the retail price \\
$w$ & Wholesale price of unit vaccine \\
$c$ & The initial demand \\
\hline
\end{tabular}

$$
\left\{\begin{array}{l}
y(t+1)=y(t)+v_{1} y(t)\left[\eta_{1}(w-c) \alpha(1-\beta p(t))-k_{1} y(t-\tau)\right] \\
p(t+1)=p(t)+v_{2} p(t)\left[\alpha(1-2 \beta p(t)+\beta w)\left(\eta_{1} y(t-\tau)+\eta_{2} s(t-\tau)\right)\right] \\
s(t+1)=s(t)+v_{3} s(t)\left[\eta_{2}(p(t)-w) \alpha(1-\beta p(t))-k_{2} s(t-\tau)\right]
\end{array}\right.
$$

The flow diagram of model (6) is as shown in Figure 2.

\section{Equilibrium Points and Hopf Bifurcation Analysis}

4.1. Positive Equilibrium Characteristic and Characteristic Equation of Model (6). We studied the equilibrium points of model (6). According to the calculations, we obtained nine equilibrium points of model (6):

$$
\begin{aligned}
& E_{1}(0,0,0) \text {, } \\
& E_{2}\left(0,0,-\frac{\alpha \eta_{2} w}{k_{2}}\right) \\
& E_{3}\left(0, \frac{1}{\beta}, 0\right) \\
& E_{4}\left(\frac{\alpha \eta_{1}(w-c)}{k_{1}}, 0,0\right) \\
& E_{5}\left(0, \frac{1+\beta w}{2 \beta}, \frac{\alpha \eta_{2}(1-\beta p)^{2}}{4 \beta k_{2}}\right) \text {, } \\
& E_{6}\left(\frac{\alpha \eta_{1}(w-c)}{k_{1}}, 0, \frac{-\alpha \eta_{2} w}{k_{2}}\right) \text {, } \\
& E_{7}\left(\frac{\alpha \eta_{1}(1-\beta p)(w-c)}{2 k_{1}}, \frac{1+\beta w}{2 \beta}, 0\right) \text {, } \\
& E_{8}\left(y_{1}^{*}, p_{1}^{*}, s_{1}^{*}\right), E_{9}\left(y_{2}^{*}, p_{2}^{*}, s_{2}^{*}\right) \text {, }
\end{aligned}
$$

$$
s_{2}^{*}=-\frac{\alpha(c-w) \eta_{1}\left[\beta k_{2} \eta_{1}^{2}(c-w)+k_{1} \eta_{2}^{2}(\beta w-1)\right]}{k_{1}^{2} \eta_{2}^{3}} .
$$

In order to analyze the stability of $E_{1} \sim E_{8}$, the Jacobian matrix of model (6) is given as follows:

$$
J=\left|\begin{array}{ccc}
A_{1} & B_{1} & 0 \\
B_{2} & A_{2} & B_{3} \\
0 & B_{4} & A_{3}
\end{array}\right|
$$

where

where 


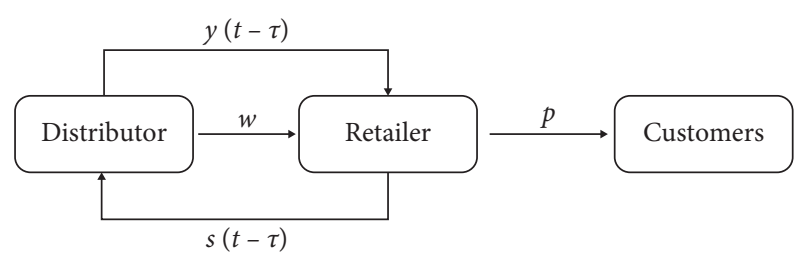

Figure 2: The flow diagram of model (6).

$$
\begin{aligned}
& A_{1}=1+v_{1} C_{1}, \\
& A_{2}=1+v_{2} C_{2}, \\
& A_{3}=1+v_{3} C_{3}, \\
& B_{1}=v_{1} y\left(\eta_{1} \alpha \beta(c-w)\right), \\
& B_{2}=v_{2} p \alpha \eta_{1}(1-2 \beta p+\beta w), \\
& B_{3}=v_{2} p \alpha \eta_{2}(1-2 \beta p+\beta w), \\
& B_{4}=v_{3} s\left(\alpha \eta_{2}(1-\beta p)-\eta_{2} \alpha \beta p-w\right), \\
& C_{1}=\eta_{1}(w-c) \alpha(1-\beta p)-2 k_{1} y, \\
& C_{2}=\alpha(1-4 \beta p+\beta w)\left(\eta_{1} y+\eta_{2} s\right), \\
& C_{3}=\eta_{2}(p-w) \alpha(1-\beta p)-2 k_{2} s .
\end{aligned}
$$

According to the eigenvalues of the Jacobian matrix evaluated at the corresponding equilibrium points, if all the nonzero eigenvalues of the Jacobian matrix are less than 1 , this equilibrium point is stable.

Take $E_{1}$ as an example. The values of $E_{1}$ are taken into Jacobian matrix $J$, and then the Jacobian matrix $J_{1}$ at the equilibrium point $E_{1}$ is acquired. Thus, $J_{1}$ can be written as

$$
J_{1}=\left|\begin{array}{ccc}
1+v_{1}\left[\eta_{1}(w-c) \alpha\right] & 0 & 0 \\
0 & 1 & 0 \\
0 & 0 & 1-v_{3} \eta_{2} w \alpha
\end{array}\right| .
$$

Obviously, the eigenvalues of $J_{1}$ are not all less than 1 , so the equilibrium point $E_{1}(0,0,0)$ is an unstable equilibrium point. Similarly, it can be proved that $E_{2} \sim E_{7}$ are unstable points.

Because of the high cost of vaccine production, $w \gg 1$ and $\beta w \gg 1$, so we can see from the calculation that $y_{1}^{*}<0$ and the values of decision variables by the decision-maker is impossible to be nonpositive in economics, which means that the equilibrium point $E_{8}$ is unstable.

Next, we analyzed the stability of the equilibrium point $E_{9}\left(y_{2}^{*}, p_{2}^{*}, s_{2}^{*}\right)$, and the characteristic polynomial of the Jacobian matrix in $E_{9}$ can be written as

$$
F(\lambda)=\lambda^{3}-T_{1} \lambda^{2}+T_{2} \lambda+T_{3}
$$

where

$$
\begin{aligned}
& T_{1}=C_{1}+C_{2}+C_{3}, \\
& T_{2}=C_{1} C_{2}+C_{1} C_{3}+C_{2} C_{3}-B_{3} B_{4}-B_{1} B_{2}, \\
& T_{3}=C_{1} B_{3} B_{4}+C_{3} B_{1} B_{2}-C_{1} C_{2} C_{3} .
\end{aligned}
$$

According to the Jury criterion, all eigenvalues of the characteristic equation are in the unit circle of the complex plane, which is a necessary and sufficient condition for the stability of the discrete system. The conditions that $E_{9}\left(y_{2}^{*}, p_{2}^{*}, s_{2}^{*}\right)$ is stable must be met as follows:

$$
\left\{\begin{array}{l}
F(1)>0, \\
F(-1)<0 \\
1>\left|T_{3}\right| \\
\left|T_{3}^{2}-1\right|>\left|-T_{3} T_{1}-T_{2}\right| .
\end{array}\right.
$$

For the sake of convenience, let $u_{1}=y(t)-y^{*}, u_{2}=$ $p(t)-p^{*}, u_{3}=s(t)-s^{*}$, and we can transform the stability of model (6) at the equilibrium point $E_{9}\left(y_{2}^{*}, p_{2}^{*}, s_{2}^{*}\right)$ into the stability at the point $(0,0,0)$ to study. Let $u_{1}=y(t), u_{2}=$ $p(t), u_{3}=s(t)$, and model (6) can be linearized at equilibrium point $E_{9}\left(y_{2}^{*}, p_{2}^{*}, s_{2}^{*}\right)$.

$$
\left\{\begin{array}{l}
y(t+1)=a_{1} y(t)+a_{2} p(t)+b_{1} y(t-\tau), \\
p(t+1)=a_{3} p(t)+b_{2} y(t-\tau)+b_{3} s(t-\tau), \\
s(t+1)=a_{4} p(t)+a_{5} s(t)+b_{4} s(t-\tau),
\end{array}\right.
$$

where

$$
\begin{aligned}
& a_{1}=1+v_{1}\left[\eta_{1}(w-c) \alpha\left(1-\beta p^{*}\right)-k_{1} y^{*}\right], \\
& a_{2}=-v_{1} y^{*} \eta_{1} \beta(w-c) \alpha, \\
& a_{3}=1+v_{2}\left[\alpha\left(1-4 \beta p^{*}+\beta w\right)\left(\eta_{1} y^{*}+\eta_{2} s^{*}\right)\right], \\
& a_{4}=v_{3} s^{*}\left[\alpha \eta_{2}(1-2 \beta p+\beta w)\right], \\
& a_{5}=1+v_{3}\left[\eta_{2}\left(p^{*}-w\right) \alpha\left(1-\beta p^{*}\right)-k_{2} s^{*}\right], \\
& b_{1}=-v_{1} y^{*} k_{1} \\
& b_{2}=v_{2} p^{*} \eta_{1}\left[\alpha\left(1-2 \beta p^{*}+\beta w\right)\right], \\
& b_{3}=v_{2} p^{*} \eta_{2}\left[\alpha\left(1-2 \beta p^{*}+\beta w\right)\right], \\
& b_{4}=-v_{3} s^{*} k_{2} .
\end{aligned}
$$

Next,

$$
\left|\begin{array}{ccc}
\lambda-a_{1}-b_{1} e^{-\lambda \tau} & -a_{2} & 0 \\
-b_{2} e^{-\lambda \tau} & \lambda-a_{3} & -b_{3} e^{-\lambda \tau} \\
0 & -a_{4} & \lambda-a_{5}-b_{4} e^{-\lambda \tau}
\end{array}\right| .
$$
(6):

$$
\begin{aligned}
f(\lambda)= & \lambda^{3}-\lambda^{2} t_{1}+\lambda t_{2}-a_{1} a_{3} a_{5} \\
& +\left[-\lambda^{2}\left(b_{1}+b_{4}\right)+\lambda t_{3}+t_{4}\right] e^{-\lambda \tau}+\left[\lambda b_{1} b_{4}+t_{5}\right] e^{-2 \widehat{\lambda} \tau},
\end{aligned}
$$

where

$$
\begin{aligned}
& t_{1}=a_{1}+a_{3}+a_{5}, \\
& t_{2}=a_{1} a_{3}+a_{1} a_{5}+a_{3} a_{5}, \\
& t_{3}=a_{1} b_{4}+a_{5} b_{1}+a_{3} b_{1}+a_{3} b_{4}-a_{4} b_{3}-a_{2} b_{2}, \\
& t_{4}=a_{1} a_{4} b_{3}+a_{2} a_{5} b_{2}-a_{1} a_{3} b_{4}-a_{3} a_{5} b_{1}, \\
& t_{5}=a_{4} b_{1} b_{3}+a_{2} b_{2} b_{4}-a_{3} b_{1} b_{4} .
\end{aligned}
$$


4.2. Case 1, $\tau=0$, Sufficient Conditions for Local Stability at Equilibrium Point $E_{9}\left(y_{2}^{*}, p_{2}^{*}, s_{2}^{*}\right)$. When $\tau=0, f(\lambda)$ can be simplified as follows:

$$
\lambda^{3}-\lambda^{2}\left(t_{1}+b_{1}+b_{4}\right)+\lambda\left(t_{2}+t_{3}+b_{1} b_{4}\right)+t_{4}+t_{5}-a_{1} a_{3} a_{5} .
$$

Let $f(\lambda)=0$; according to Routh-Hurwitz criterion, if $\left(H_{1}\right):-\left(t_{1}+b_{1}+b_{4}\right)>0,\left(t_{2}+t_{3}+b_{1} b_{4}\right)>0,-\left(t_{1}+b_{1}+\right.$ $\left.b_{4}\right)\left(t_{2}+t_{3}+b_{1} b_{4}\right)>\left(t_{4}+t_{5}-a_{1} a_{3} a_{5}\right)$ and $\left(t_{4}+t_{5}-\right.$ $\left.a_{1} a_{3} a_{5}\right)>0$, hold, then model (6) is asymptotically stable at the equilibrium point.

4.3. Case 2, $\tau>0$, Sufficient Conditions for Local Stability at Equilibrium Point $E_{9}\left(y_{2}^{*}, p_{2}^{*}, s_{2}^{*}\right)$. Let $f(\lambda)=0$ and multiply both sides by $e^{\lambda \tau}$, and then

$$
\begin{aligned}
& -\lambda^{2}\left(b_{1}+b_{4}\right)+\lambda t_{3}+t_{4}+\left(\lambda^{3}-\lambda^{2} t_{1}+\lambda t_{2}-a_{1} a_{3} a_{5}\right) e^{\lambda \tau} \\
& +\left[\lambda b_{1} b_{4}+t_{5}\right] e^{-\lambda \tau}=0 .
\end{aligned}
$$

Assume that $\lambda=i \omega(\omega>0)$ is the root of (21); then,

$$
\left\{\begin{array}{l}
\Delta_{1} \cos (\omega \tau)+\Delta_{2} \sin (\omega \tau)=-\omega t_{3} \\
\Delta_{3} \cos (\omega \tau)+\Delta_{4} \sin (\omega \tau)=-\omega^{2}\left(b_{1}+b_{4}\right)-t_{4}
\end{array}\right.
$$

where

$$
\begin{gathered}
\Delta_{1}=-\omega^{3}+\omega b_{1} b_{4}+\omega t_{2}, \\
\Delta_{2}=\omega^{2} t_{1}-a_{1} a_{3} a_{5}-t_{5}, \\
\Delta_{3}=\omega^{2} t_{1}-a_{1} a_{3} a_{5}+t_{5}, \\
\Delta_{4}=\omega^{3}+\omega b_{1} b_{4}-\omega t_{2} .
\end{gathered}
$$

By the aid of (22), we can obtain

$$
\left\{\begin{array}{l}
\sin (\omega \tau)=\frac{\left[-\omega^{2}\left(b_{1}+b_{4}\right)-t_{4}\right] \Delta_{1}+\omega t_{3} \Delta_{3}}{\Delta_{1} \Delta_{4}-\Delta_{2} \Delta_{3}}, \\
\cos (\omega \tau)=\frac{-\omega t_{3} \Delta_{4}+\left[\omega^{2}\left(b_{1}+b_{4}\right)+t_{4}\right] \Delta_{2}}{\Delta_{1} \Delta_{4}-\Delta_{2} \Delta_{3}} .
\end{array}\right.
$$

By the aid of (24), we have

$$
\omega^{12}+\omega^{10} r_{1}+\omega^{8} r_{2}+\omega^{6} r_{3}+\omega^{5} r_{4}+\omega^{4} r_{5}+\omega^{2} r_{6}+r_{7}=0
$$

where

$$
\begin{aligned}
r_{1}= & \left(b_{1}+b_{4}\right)^{2}+\left(2 t_{1}^{2}-4 t_{2}\right), \\
r_{2}= & 6 t_{2}^{2}-2\left(b_{1} b_{4}\right)^{2}+t_{1}^{4}-4 t_{1} a_{1} a_{3} a_{5}-t_{1}^{2}\left(b_{1}+b_{4}\right)^{2}-t_{3}^{2} \\
& +\left(2 t_{1} t_{3}-2 t_{4}\right)\left(b_{1}+b_{4}\right)+\left(2 b_{1} b_{4}+2 t_{2}\right)\left(b_{1}+b_{4}\right)^{2}, \\
r_{3}= & \left(b_{1} b_{4}+t_{2}\right)^{2}\left(2 b_{1} b_{4}-2 t_{2}\right)-\left(b_{1} b_{4}-t_{2}\right)^{2}\left(2 b_{1} b_{4}+2 t_{2}\right) \\
& -4 t_{1}^{3} a_{1} a_{3} a_{5}-2\left(a_{1} a_{3} a_{5}\right)^{2}+2 t_{5}^{2}-t_{3}^{2}\left(2 b_{1} b_{4}-2 t_{2}\right)-2 t_{1}^{2} t_{4}\left(b_{1}+b_{4}\right)+2 t_{1}\left(b_{1}+b_{4}\right)^{2}, \\
r_{4}= & 2 t_{1} t_{3}\left(b_{1}+b_{4}\right), \\
r_{5}= & \left(b_{1} b_{4}+t_{2}\right)^{2}\left(b_{1} b_{4}-t_{2}\right)^{2}+2 t_{1}^{2}\left(a_{1} a_{3} a_{5}\right)^{2}+t_{5}^{2}+4 t_{1}^{2}\left(\left(a_{1} a_{3} a_{5}\right)^{2}-t_{5}^{2}\right) \\
& -t_{3}^{2}\left(b_{1} b_{4}-t_{2}\right)^{2}+t_{1}^{2} t_{4}^{2}+\left(a_{1} a_{3} a_{5}+t_{5}\right)^{2}\left(b_{1}+b_{4}\right)^{2}+t_{4}^{2}\left(2 b_{1} b_{4}+2 t_{2}\right), \\
r_{6}= & -2 t_{1}\left(a_{1} a_{3} a_{5}+t_{5}\right)^{2}\left(a_{1} a_{3} a_{5}-t_{5}\right)-2 t_{1}\left(a_{1} a_{3} a_{5}-t_{5}\right)^{2}\left(a_{1} a_{3} a_{5}+t_{5}\right) \\
& +2 t_{1} t_{4}^{2}\left(a_{1} a_{3} a_{5}+t_{5}\right)^{2}-t_{4}^{2}\left(b_{1}+b_{4}\right)^{2}, \\
r_{7}= & \left(a_{1} a_{3} a_{5}+t_{5}\right)^{2}\left(a_{1} a_{3} a_{5}-t_{5}\right)^{2} .
\end{aligned}
$$

Define $g(\omega)=\omega^{12}+\omega^{10} r_{1}+\omega^{8} r_{2}+\omega^{6} r_{3}+\omega^{5} r_{4}+\omega^{4} r_{5}+$ $\omega^{2} r_{6}+r_{7}$. Without loss of generality, we assume that $\left(H_{2}\right): g(\omega)$ has at least one positive real root. In order to establish the main results of this article, we assume $g(\omega)$ has $n$ positive roots, denoted by $g_{1}, g_{2}, \ldots, g_{n}, 0<n \leq 12$. From (24), we can get

$$
\tau_{k}^{(j)}=\frac{1}{\omega_{k}} \arccos \left\{\frac{-\omega t_{3} \Delta_{4}+\left[\omega^{2}\left(b_{1}+b_{4}\right)+t_{4}\right] \Delta_{2}}{\Delta_{1} \Delta_{4}-\Delta_{2} \Delta_{3}}\right\}+\frac{2 j \pi}{\omega_{k}}, \quad k=1,2,3, \ldots, n ; j=0,1, \ldots
$$




$$
\tau_{0}=\min \left\{\tau_{k}^{(j)} \mid k=1,2,3, \ldots, n ; j=0.1, \ldots\right\}=\min \left\{\tau_{k}^{(0)} \mid k=1,2,3, \ldots, n\right\}=\tau_{k_{0}}^{(0)}
$$

Differentiating both sides of (21) with regard to $\tau$, we can get

$$
\left[\frac{\mathrm{d} \lambda}{\mathrm{d} \tau}\right]^{-1}=\frac{-2 \lambda\left(b_{1}+b_{4}\right)+t_{3}+\left(3 \lambda^{2}-2 \lambda t_{1}+t_{2}\right) e^{\lambda \tau}+b_{1} b_{4} e^{-\lambda \tau}}{\lambda e^{\lambda \tau}\left(\lambda^{3}-\lambda^{2} t_{1}+\lambda t_{2}-a_{1} a_{3} a_{5}\right)-\lambda e^{-\lambda \tau}\left(\lambda b_{1} b_{4}+t_{5}\right)}-\frac{\tau}{\lambda}
$$

When $\tau=\tau_{0}$ and substituting $\lambda=i \omega_{0}$ into (31), it can be achieved that

$$
\operatorname{Re}\left[\frac{\mathrm{d} \lambda}{\mathrm{d} \tau}\right]_{\tau=\tau_{0}}^{-1}=\frac{X_{1} X_{2}+Y_{1} Y_{2}}{X_{1}^{2}+Y_{1}^{2}}
$$

where

$$
\begin{aligned}
& X_{1}=\left(\omega_{0}^{4}-\omega_{0}^{2} t_{2}+\omega_{0}^{2} b_{1} b_{4}\right) \cos \left(\omega_{0} \tau_{0}\right)+\left(-\omega_{0}^{3} t_{1}+\omega_{0} a_{1} a_{3} a_{5}-\omega_{0} t_{5}\right) \sin \left(\omega_{0} \tau_{0}\right), \\
& Y_{1}=\left(\omega_{0}^{3} t_{1}-\omega_{0} a_{1} a_{3} a_{5}-\omega_{0} t_{5}\right) \cos \left(\omega_{0} \tau_{0}\right)+\left(\omega_{0}^{4}-\omega_{0}^{2} t_{2}-\omega_{0}^{2} b_{1} b_{4}\right) \sin \left(\omega_{0} \tau_{0}\right), \\
& X_{2}=\left(-3 \omega_{0}^{2}+t_{2}+b_{1} b_{4}\right) \cos \left(\omega_{0} \tau_{0}\right)+2 \omega_{0} t_{1} \sin \left(\omega_{0} \tau_{0}\right)+t_{3}, \\
& Y_{2}=-2 \omega_{0} t_{1} \cos \left(\omega_{0} \tau_{0}\right)+\left(-3 \omega_{0}^{2}+t_{2}-b_{1} b_{4}\right) \sin \left(\omega_{0} \tau_{0}\right)-2 \omega_{0}\left(b_{1}+b_{4}\right) .
\end{aligned}
$$

To ensure the condition of the occurrence for Hopf bifurcation, we have the following hypothesis $\left(H_{3}\right): X_{1} X_{2}+Y_{1} Y_{2} \neq 0$, and then we have the following results.

Theorem 1. For model (6), if the conditions $\left(H_{1}\right),\left(H_{2}\right)$, and $\left(\mathrm{H}_{3}\right)$ hold, the equilibrium point $E_{9}\left(y_{2}^{*}, p_{2}^{*}, s_{2}^{*}\right)$ is asymptotically stable when $\tau \in\left[0, \tau_{0}\right)$; when $\tau=\tau_{0}$, model (6) undergoes a Hopf bifurcation at equilibrium point $E_{9}\left(y_{2}^{*}, p_{2}^{*}, s_{2}^{*}\right)$; and model (6) is unstable at equilibrium point $E_{9}\left(y_{2}^{*}, p_{2}^{*}, s_{2}^{*}\right)$ when $\tau>\tau_{0}$.

\section{Numerical Simulation}

In order to further verify the obtained theoretical results, two-dimensional bifurcation diagram is used to analyze the influence of the time-delay and adjustment speed of decision variables on the stability of the system. We use the largest Lyapunov exponent and entropy to measure system complexity, respectively. The principle of the largest Lyapunov exponent is that when exponent value is less than zero the system is stable; in contrast, when the exponent value is greater than zero, the system is unstable. The rules of entropy to judge system complexity is as follows: the system is in a stable state when the entropy is low; in contrast, the system is in chaos when the entropy is large.

5.1. Hopf Bifurcation Diagram Caused by Delay. We set the parameters as follows: $\alpha=1, \beta=0.29, p=200, w=100, c=$ $50, \eta_{1}=0.6, \eta_{2}=0.3, k_{1}=0.1, k_{2}=0.4$ and by some computations we obtained $\tau_{0}=0.304, \omega_{0}=1.68$. First, the twodimensional bifurcation diagram of the system stability domain changes with the time-delay as shown in Figure 3(a).

As can be seen from Figure 3(a), model (6) gradually produces Hopf bifurcation from stability with the increase of $\tau$ and then changes to unstable state, and the critical point of bifurcation is $\tau_{0}=0.304$. When $\tau<0.304$, equilibrium point
$E_{9}\left(y_{2}^{*}, p_{2}^{*}, s_{2}^{*}\right)$ is asymptotically stable; and when $\tau>0.304$, model (6) goes from Hopf bifurcation to the unstable state at equilibrium point $E_{9}\left(y_{2}^{*}, p_{2}^{*}, s_{2}^{*}\right)$.

From Figure 3(a), it has been shown that the vaccine supply chain equilibrium is locally asymptotically stable if the time-delay parameter below the critical value. The advantage of a stable state is that the decision-makers in the system generally know the development law of the decision variables; at this time, the decision-maker can control the vaccine transportation more easily. However, once the time-delay parameter exceeds a certain threshold, the system undergoes a Hopf bifurcation and goes into chaos. The emergence of Hopf bifurcation means that model (6) changes from the vaccines transportation equilibrium to a limit cycle. At this time, the vaccine transportation is out of control. Figure 3(b) shows the largest Lyapunov exponent when $\tau$ increases. Comparing Figure 3(a) with Figure 3(b), when bifurcation occurs and the system gets in chaos, it can be seen that the largest Lyapunov exponents are consistent with the bifurcation diagram shown in Figure 3(a). Similarly, comparing Figure 3(a) with Figure 3(c), it can be seen that the entropy of the system is increasing rapidly when bifurcation occurs and gets in chaos.

5.2. Bifurcation Diagram Caused by Adjusting Speed of Decision Variable. In this paper, we show the dynamic characteristics of the system in four ways: bifurcation diagram, maximum Lyapunov exponent, entropy, and chaos attractor.

5.2.1. Chaos and Bifurcation Phenomenon. When $\tau=0.1$ and other parameters remain unchanged, the influence of time-delay on the stability of the system can be eliminated. Assume $v_{2}=0.7$, and $v_{3}=0.6$, and the value of $v_{1}$ ranges from 0 to 0.5 . We examine the effect of the adjusting speed $v_{1}$ 


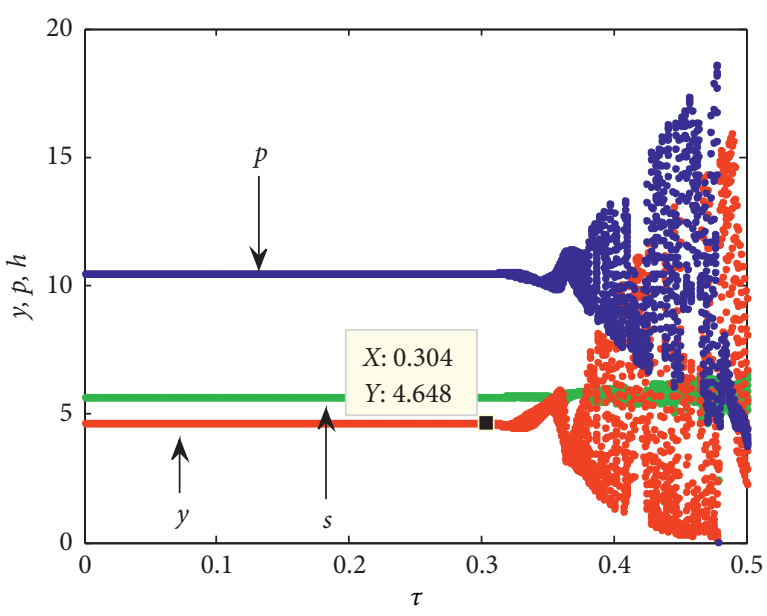

(a)

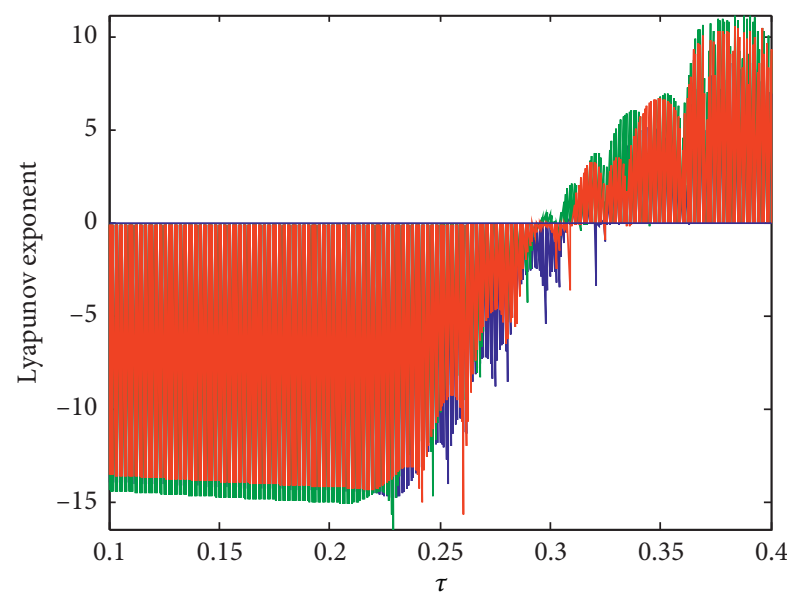

(b)

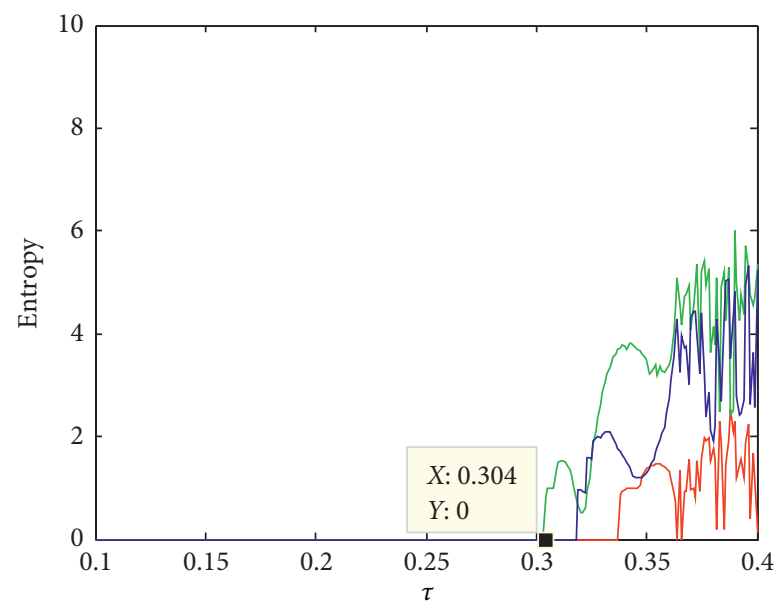

(c)

Figure 3: The impact of $\tau$ on the stability and complexity of model (6). (a) Bifurcation diagram of model (6) with (b) Lyapunov exponents with respect to $\tau \tau$ of Figure 3(a). (c) The entropy diagram of Figure 3(a).

of the cold chain transportation level on the system stability. Figure 4(a) demonstrates that when $v_{1}$ increases from 0 to 0.251 , the cold chain transportation level $y$, retail price of unit vaccine $p$, and inspection level $s$ are stable at 5.2, 2.472, and 1.837 , respectively; by contrast, when $v_{1}$ increases to 0.251 , the system turns into stable cycles of period 2, which means that each decision's variables have two possible values; when $v_{1}>0.38$, the cold chain transportation level $y$, retail price of unit vaccine $p$, and inspection level $s$ have a lot of possible values.

Ceteris paribus, assuming that $v_{1}=0.45$, and $v_{3}=0.32$, Figure 5 presents the effect of the adjustment speed $v_{2}$ of retail price of vaccine on system stability. When $v_{2}$ changes at $[0,0.5]$, the changes of decision variables are shown in Figure 5(a). When $v_{2} \in[0,0.166)$, the cold chain transportation level $y$, retail price of unit vaccine $p$, and inspection level $s$ are stable at 1.148, 1.627, and 2.809, respectively; when $v_{2}$ increases to 0.166 , the first bifurcation occurs, and the system turns into stable cycles of period 2 , which means that each decision variables have two possible values; with the further increase of $v_{2}$, the cold chain transportation level $y$, retail price of unit vaccine $p$. and inspection level $s$ become chaotic; that is, each decision variables have a lot of possible values.

Furthermore, assuming that $v_{1}=0.19$ and $v_{2}=0.36$, Figure 6 analyzes the effect of $v_{3}$ on system stability. When $v_{3}$ changes at $[0,0.5]$, the changes of decision variables are shown in Figure 6(a). When $v_{3} \in[0,0.151)$, the cold chain transportation level $y$, retail price of unit vaccine $p$, and inspection level $s$ are stable at 2.492, 1.814, and 1.844, respectively; when $v_{3}$ increases to 0.151 , the system turns into stable cycles of period 2; with the increase of $v_{3}$, eventually the cold chain transportation level $y$, retail price of unit vaccine $p$, and inspection level $s$ become chaotic; that is, each decision's variables have a lot of possible values.

From Figures 4(a)-6(a), it can be seen that as the adjustment speed of decision variables increases, the number of system cycles increases, and finally the chaotic state appears. The increasing of adjustment speed of decision variables means that there are irrational decisions in the vaccine supply chain. It is difficult to obtain the sustainable development of the vaccine supply chain structure. 


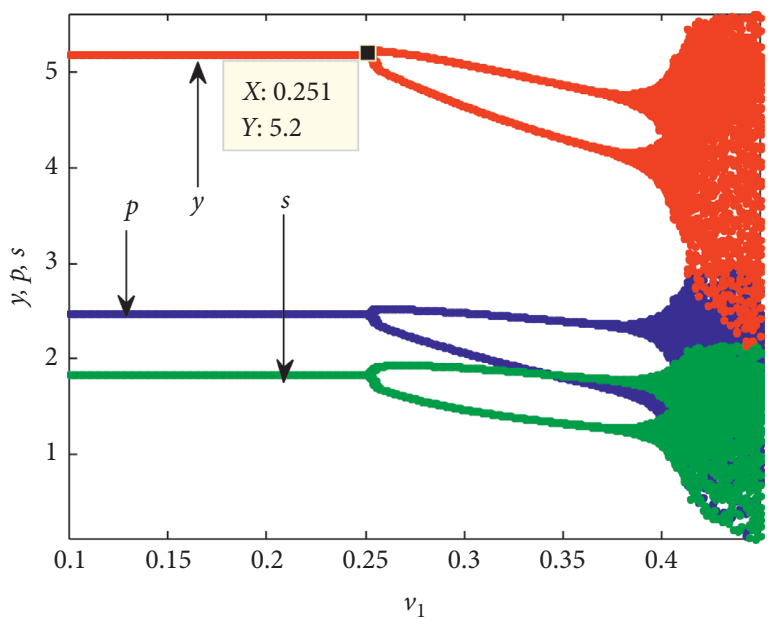

(a)

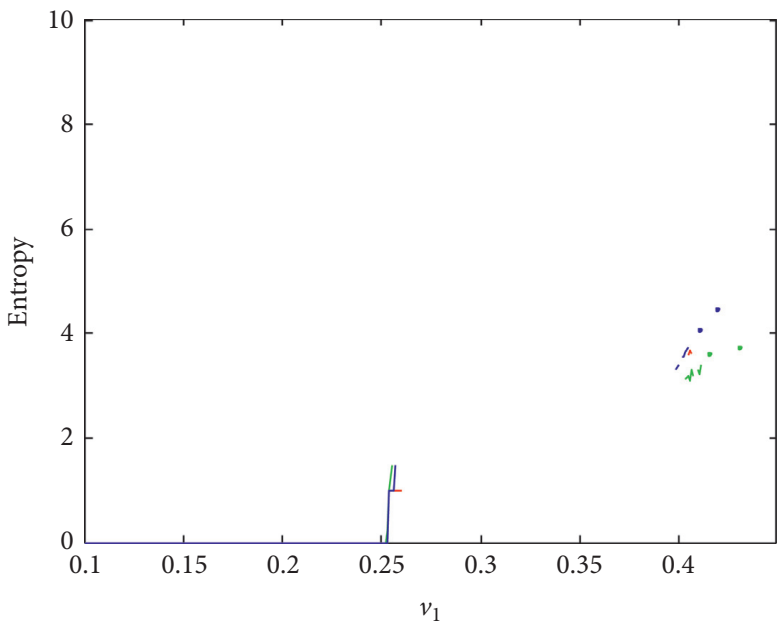

(c)

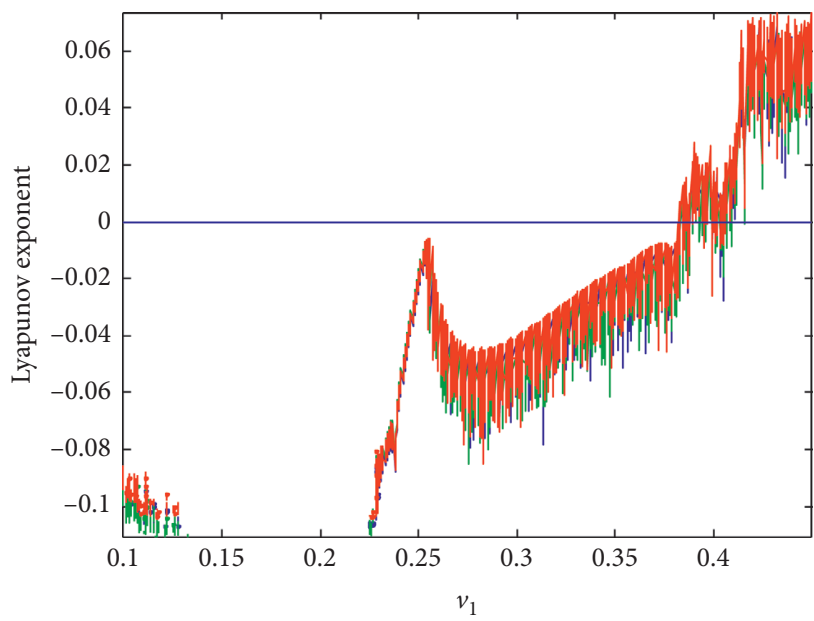

(b)

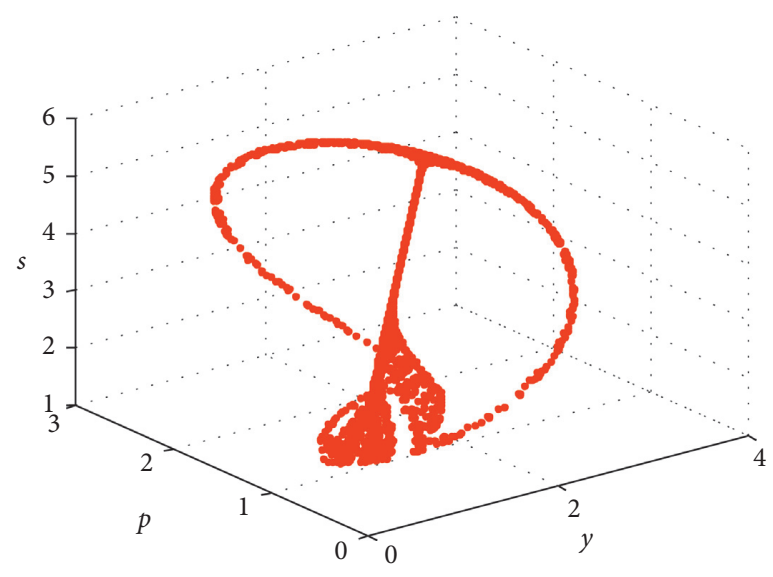

(d)

Figure 4: Dynamic evolution of decision variables with $v_{1}$. (a) $v_{2}=0.7, v_{3}=0.6$. (b) Lyapunov exponents for $v_{1}$ varying from 0 to 0.5 of Figure 4(a). (c) The entropy diagram of Figure 4(a). (d) Chaotic attractor of the system.

5.2.2. Largest Lyapunov Exponent. The largest Lyapunov exponent can be used to determine whether the system is in a chaotic state. If the largest Lyapunov exponent is less than 0 , the system is in a stable state; if the largest Lyapunov exponent is close to 0 , the system is in a periodic motion; if the largest Lyapunov exponent is greater than 0 , the system is in a chaotic state. Take Figure 4(b) for example; when the largest Lyapunov exponent first returns to the 0 axis, the system reaches two times periodic bifurcation point. When the system returns for the second time and passes through the 0 axis, the system enters a chaotic state. Comparing the largest Lyapunov exponent with Figure 4(a), when bifurcation occurs and the system becomes in chaos, it can be seen that the largest Lyapunov exponents are consistent with the bifurcation diagram shown in Figure 4(a). Figures 5(b)6(b) show similar results, which are not repeated here.

5.2.3. Entropy. When many scholars analyze the influence of time-delay parameter in the system, they only prove and give the conditions for the stability of the system. However, there are few people discussing what the chaotic behavior will bring to the system. The essence of entropy is the internal chaos of a system. According to information theory, the higher the entropy is, the higher the chaos of the system's internal information is, which also results in the decisionmaker making more effort to acquire useful information. From Figure $4(\mathrm{c})$, we can know that when $v_{1} \in(0,0.251)$, the entropy of model (6) is equal to 0 , and the cold chain transportation level $y$, retail price of unit vaccine $p$, or inspection level $s$ has a unique value. When $v_{1}>0.251$, model (6) undergoes a doubly periodic bifurcation state and falls into chaos finally, the entropy of model (6) continues to increase, and the cold chain transportation level $y$, retail price of unit vaccine $p$, or inspection level $s$ becomes unstable and has lots of values. From the information theory, the large entropy can increase uncertainty of information on decision variables, which may cause the distributor and retailer to miss the best decision-making opportunity to maintain vaccines' activity in the process of vaccine transportation. Figures 5(c)-6(c) show similar results. 


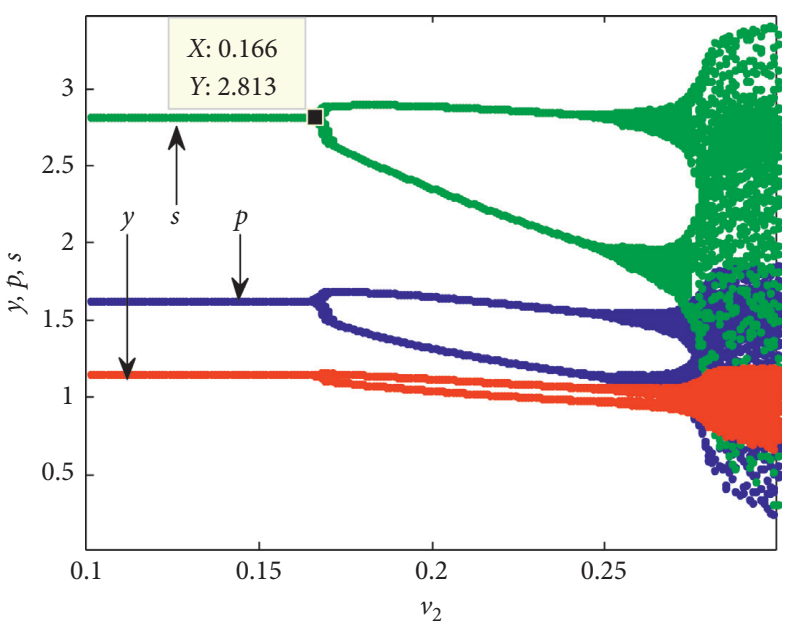

(a)

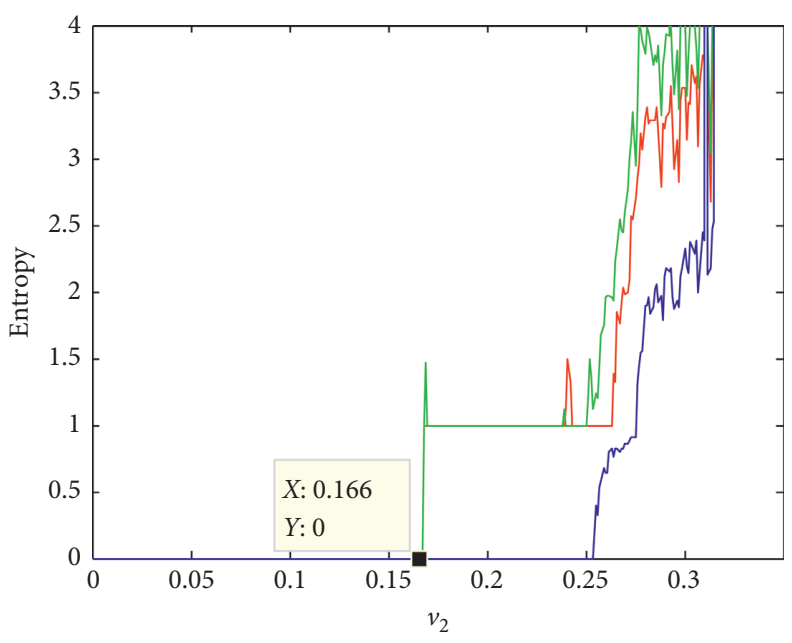

(c)

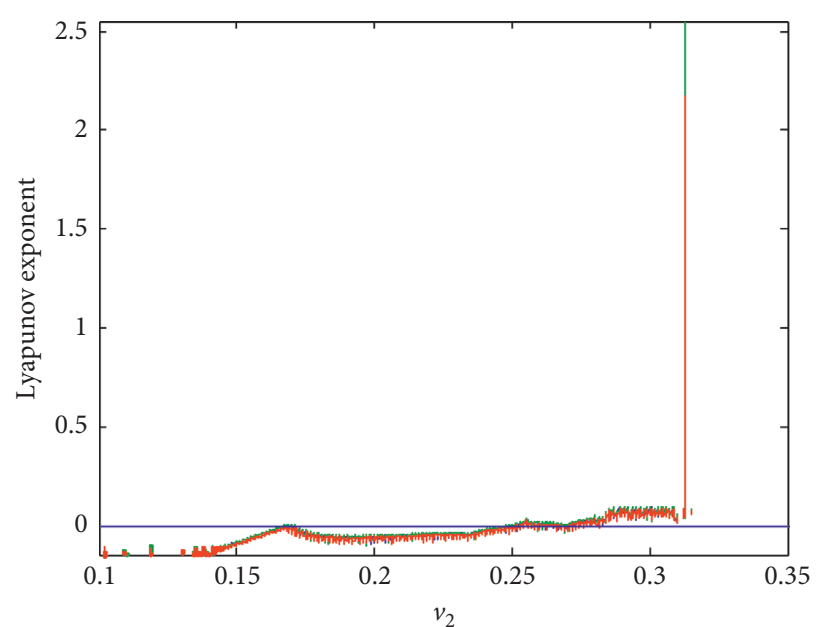

(b)

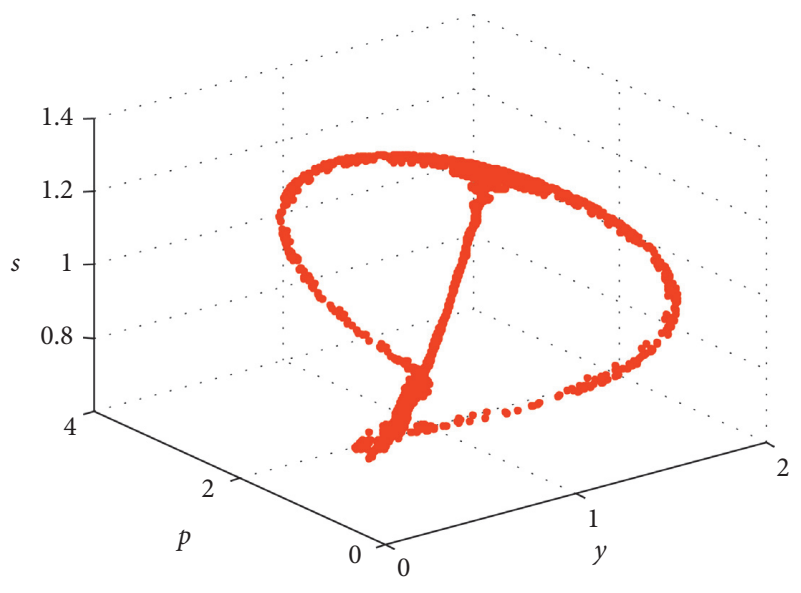

(d)

Figure 5: Dynamic evolution of decision variables with $v_{2}$. (a) $v_{1}=0.45, v_{3}=0.32$. (b) Lyapunov exponents for $v_{2}$ varying from 0 to 0.5 of Figure 5(a). (c) The entropy diagram of Figure 5(a). (d) Chaotic attractor of the system.

5.2.4. Chaotic Attractor. The chaotic attractors are shown in Figures 4(d)-6(d) and are analyzed. When the system goes into a chaotic state, the structure of the chaotic attractor can be more complicated. Take Figure 4(d) for example; when $v_{1}>0.251$, the system is in a chaotic state, and the chaotic attractor is complicated. Figures 5(d)-6(d) show similar results.

\subsection{Influence of Adjustment Speed of Decision Variables on} Stability Critical Point. Compared with Figure 4(a), Figure $7(\mathrm{a})$ shows the change of system stability with $v_{1}$ when $v_{3}$ is constant and $v_{2}$ is reduced to 0.3 . Obviously, with the decrease of retailer's adjustment speed $v_{2}$, the critical point of stability moves to the right. Figure $7(\mathrm{~b})$ shows the change of system stability with $v_{1}$ when $v_{2}$ is constant and $v_{3}$ is reduced to 0.27 . In the same way, with the decrease of retailer's adjustment speed $v_{3}$, the critical point of the stability moves to the right. It is not hard to see that, in terms of the goal of affecting the critical point of the stable state of the vaccine supply chain, there exist reverse changes in the relationship between the adjustment speed of the distributor's decision variable $v_{1}$ and the adjustment speed of the retailer's decision variable $v_{2}$ and $v_{3}$. This interesting conclusion can imply powerful suggestions for distributor and retailer to find a balance in the process of vaccines transportation.

\section{Chaos Control}

In the vaccine supply chain especially, chaos can make the vaccines transportation difficult to be carried out smoothly, which inactivates the vaccines. In order to avoid chaos, we try to control the chaos by adjusting the parameter control method and variable feedback control method, respectively. The chaotic control effects of adjusting parameters on model (6) are demonstrated by numerical simulation. As can be seen from the foregoing, let $\tau=0.5, v_{1}=0.4, v_{2}=v_{3}=0.5$. When other parameters keep fixed, the system is in a chaotic state. 


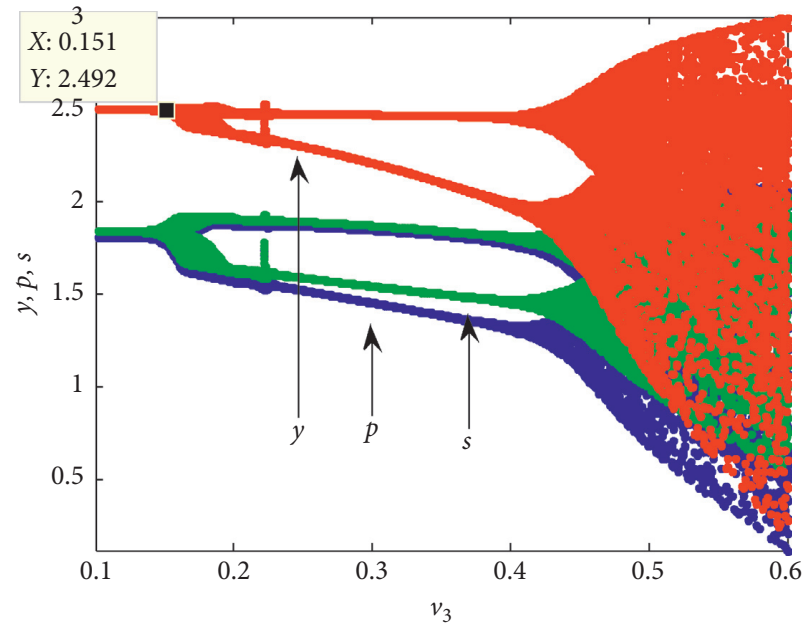

(a)

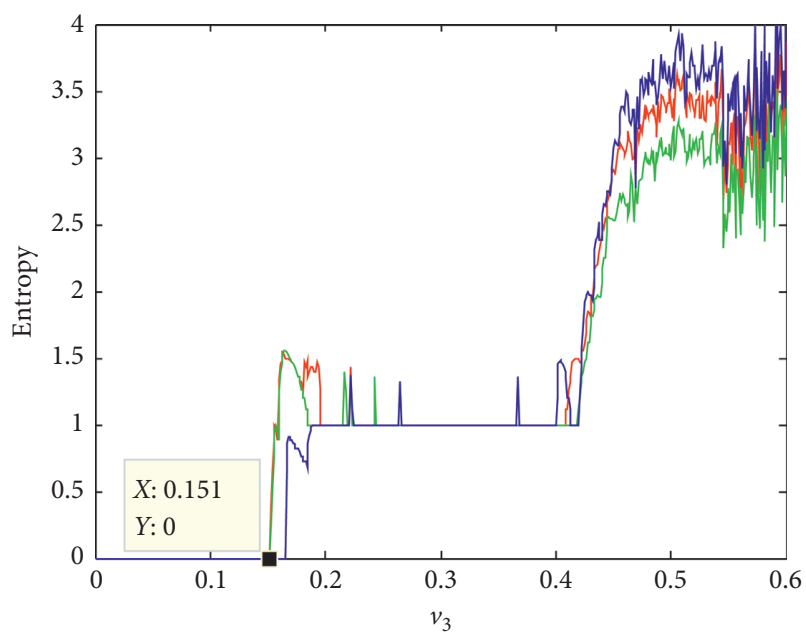

(c)

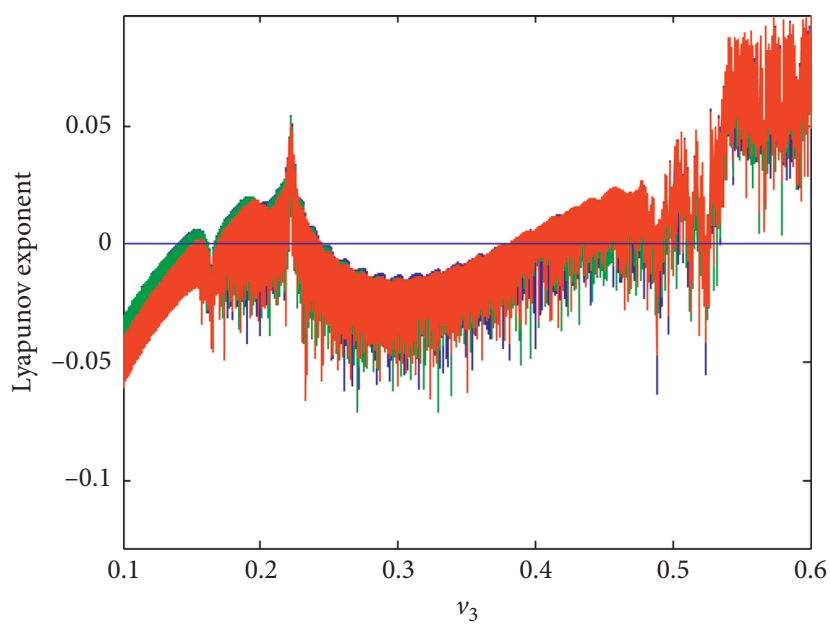

(b)

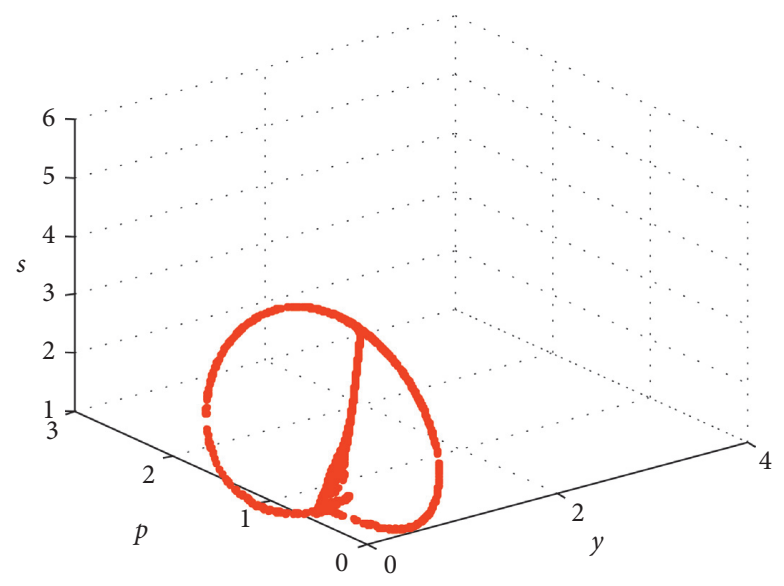

(d)

Figure 6: Dynamic evolution of decision variables with $v_{3}$. (a) $v_{1}=0.19, v_{3}=0.36$. (b) Lyapunov exponents for $v_{3}$ varying from 0 to 0.5 of Figure 6(a). (c) The entropy diagram of Figure 6(a). (d) Chaotic attractor of the system.

6.1. Adjustment Parameter Control Method. The control methods are mainly divided into feedback control method and nonfeedback control method. The adjusting parameter control method is one of the feedback control methods. This method is to change the internal structure of the original system to achieve control effect. The original system is as follows:

$$
\left\{\begin{array}{l}
y(t+1)=y(t)+v_{1} y(t)\left[\eta_{1}(w-c) \alpha(1-\beta p(t))-k_{1} y(t-\tau)\right], \\
p(t+1)=p(t)+v_{2} p(t)\left[\alpha(1-2 \beta p(t)+\beta w)\left(\eta_{1} y(t-\tau)+\eta_{2} s(t-\tau)\right)\right] \\
s(t+1)=s(t)+v_{3} s(t)\left[\eta_{2}(p(t)-w) \alpha(1-\beta p(t))-k_{2} s(t-\tau)\right] .
\end{array}\right.
$$




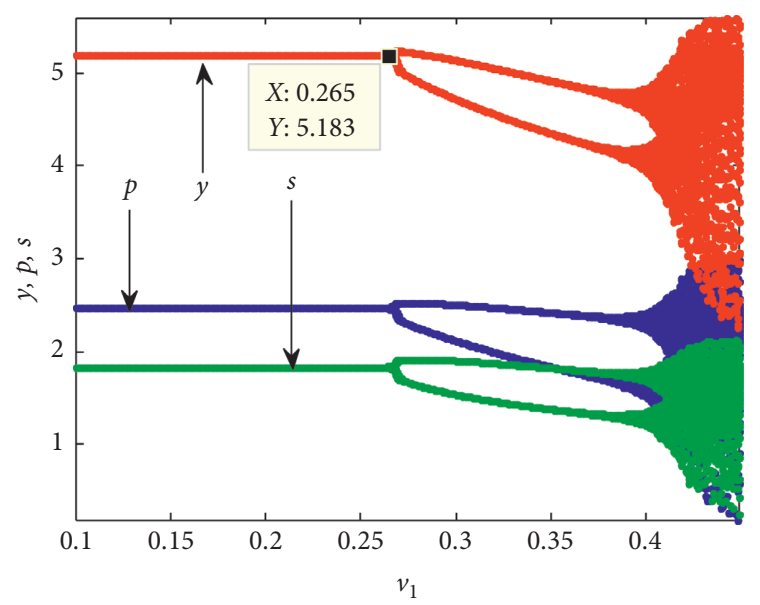

(a)

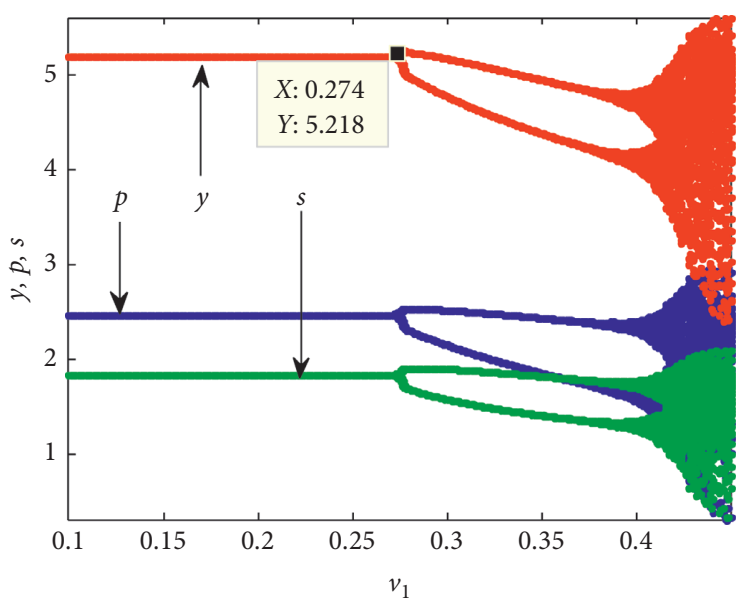

(b)

FIgURe 7: Dynamic evolution of decision variables with $v_{1}$. (a) $v_{2}$ is reduced to 0.3 . (b) $v_{3}$ is reduced to 0.27 .

The system is transformed into the following one by parameter adjustment control [37]:

$$
\left\{\begin{array}{l}
y(t+1)=(1-u)\left\{y(t)+v_{1} y(t)\left[\eta_{1}(w-c) \alpha(1-\beta p(t))-k_{1} y(t-\tau)\right]\right\}+u y(t), \\
p(t+1)=(1-u)\left\{p(t)+v_{2} p(t)\left[\alpha(1-2 \beta p(t)+\beta w)\left(\eta_{1} y(t-\tau)+\eta_{2} s(t-\tau)\right)\right]\right\}+u p(t) \\
s(t+1)=(1-u)\left\{s(t)+v_{3} s(t)\left[\eta_{2}(p(t)-w) \alpha(1-\beta p(t))-k_{2} s(t-\tau)\right]\right\}+u s(t) .
\end{array}\right.
$$

With the change of the added adjustment parameter, the system changes as shown in Figure 8. When $u=0$, the system is in a chaotic state, which indicates that the distributor and the retailer cannot make joint decisions to control chaos. With the increase of $u$, the system reaches a stable state from chaotic state, which indicates that the distributor and retailer can effectively control the chaos by taking joint measures, such as signing contracts and so on.

6.2. State Variable Feedback Control Method. The state variable feedback control is a characteristic of modern control theory. State variable feedback control means that each state variable of the system is multiplied by the corresponding feedback coefficient and fed back to the input end and added to the reference input, and the sum is used as the control signal of the controlled system. The state variables of a system can show the internal characteristics of the whole system without knowing the internal structure of the system, and the control signals can be collected from the whole space or from the local. Therefore, compared with the traditional feedback control, the state variable feedback control can not only be a more excellent and effective control method, but also the form of its controller is more abundant [38].

$$
\left\{\begin{array}{l}
y(t+1)=y(t)+v_{1} y(t)\left[\eta_{1}(w-c) \alpha(1-\beta p(t))-k_{1} y(t-\tau)\right]-u y(t) \\
p(t+1)=p(t)+v_{2} p(t)\left[\alpha(1-2 \beta p(t)+\beta w)\left(\eta_{1} y(t-\tau)+\eta_{2} s(t-\tau)\right)\right]-u p(t) \\
s(t+1)=s(t)+v_{3} s(t)\left[\eta_{2}(p(t)-w) \alpha(1-\beta p(t))-k_{2} s(t-\tau)\right]-u s(t)
\end{array}\right.
$$

Figure 9 shows that the chaotic system gradually returns to a stable state through variable feedback control method. When $u=0$, the system is in a chaotic state, which indicates that the government does not take effective control measures for chaotic systems at this time. When $u>0.253$, the system returns to a stable state, which indicates that the government takes external intervention measures to accelerate the system to a stable state and ensure stable economic development.

From the comparison between Figures 8 and 9, it is obvious that the control system in Figure 9 enters the stable state earlier than in Figure 8, which means that the 


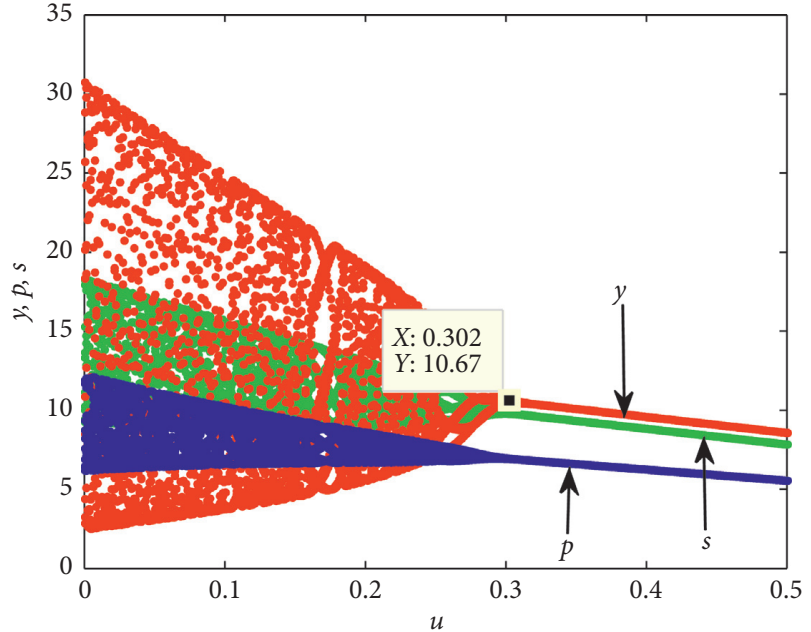

FIGURE 8: Systematic bifurcations with variation of adjustment parameter $u$.

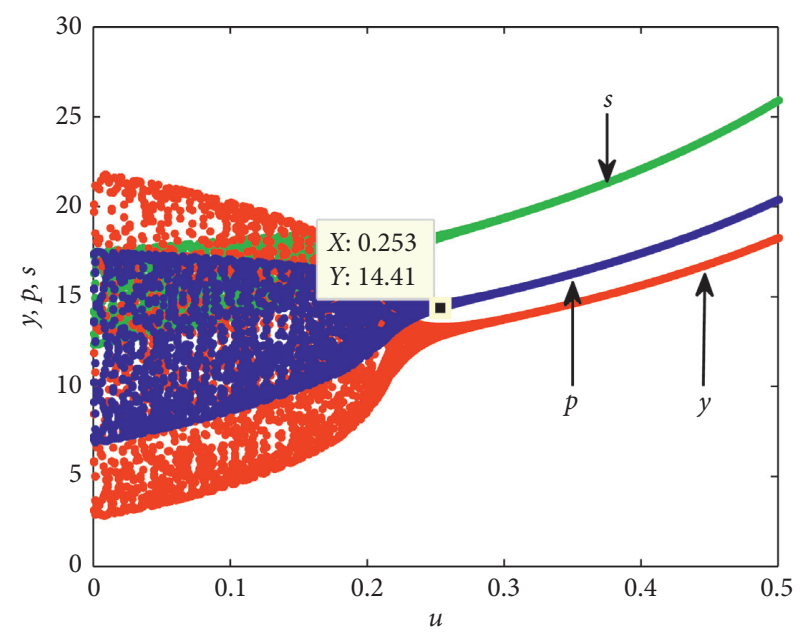

FIGURE 9: Systematic bifurcations with variation of adjustment parameter $u$.

control effect of the variable feedback control method is better than that of adjusting parameter control method. The reason is that the structure of cold chain transportation in the vaccine supply chain is complex, and chaos control can bring about extra cost. It is difficult for the distributor and retailer to control the chaotic vaccine supply chain. At this time, it is imperative that external forces are used to control the chaos in the vaccine supply chain.

\section{Conclusion}

In this study, we focus on the vaccine supply chain composed of a distributor and retailer. We assume that the distributor chooses cold chain transportation to ensure vaccines activity, and the retailer checks whether the cold chain transportation effect that the distributor chose is qualified each time. We thought that the decisions of the distributor and retailer are not instantaneous and established a decision-making timedelay model. By using two-dimensional bifurcation diagram,
Largest Lyapunov exponent, and entropy and chaotic attractors, we not only acquired the condition that the timedelay causes the system to produce a Hopf bifurcation, but also the condition that the adjustment speed of the decision variable causes the system to produce a period-doubling bifurcation. In addition, we used two different methods to control the chaotic system and compared the control effect. We drew the following conclusions.

(1) When time-delay parameter $\tau \in\left[0, \tau_{0}\right)$, the vaccines transportation equilibrium is locally asymptotically stable. At this time, the distributor and retailer can cooperate well to ensure vaccines' activity. When time-delay parameter $\tau \geq \tau_{0}$, the vaccine transportation system produces Hpof bifurcation and loses stability, which means that the vaccine supply chain changes from the vaccines transportation equilibrium to a limit cycle.

(2) If the distributor and retailer adjust the decision variables too quickly, the vaccine supply chain will bifurcate and fall into chaos and the entropy of the system will increase, which will cause the decisionmaker to collect more additional information to find out the appropriate results, and it may make the decision-maker miss the best time to keep the vaccines active.

(3) In terms of the goal of affecting the critical point of the stable state of the vaccine supply chain, there exist reverse changes in the relationship between the adjustment speed of the distributor's decision variable $v_{1}$ and the adjustment speed of the retailer's decision variable $v_{2}$ (or $\left.v_{3}\right)$.

(4) Compared with the internal joint control of the distributor and retailer, external control, such as government intervention, will have a better control effect of the chaos of the system.

In order to simplify the problem, we established a simplified vaccine supply chain model, while ignoring many important features of the vaccine supply chain. For example, there may be some loss of vaccines during transportation. A supply chain considering the wastage rate of vaccines will make the model closer to reality. Also, vaccines are different from ordinary profitable products, and a supply chain considering social responsibility is also a research hotspot. We will study these issues in the future.

\section{Data Availability}

The data used to support the findings of this study are available from the corresponding author upon request.

\section{Conflicts of Interest}

The authors declare no conflicts of interest.

\section{Acknowledgments}

This research was supported by the National Natural Science Foundation of China (no. 31601224), Key Project of Natural 
Science Research of Universities in Anhui Province (KJ2019A0662), and the Graduate Research and Innovation Fund of Anhui University of Finance and Economics (ACYC2019205).

\section{References}

[1] Y.W. Yang, B. Hoda, and R. Jayant, "Optimizing vaccine distribution networks in low and middle-income countries," Omega, 2020, In press.

[2] M. K. Patel, L. Dumolard, Y. Nedelec et al., "Progress toward regional measles elimination-worldwide, 2000-2018," MMWR. Morbidity and Mortality Weekly Report, vol. 68, no. 48, pp. 1105-1111, 2019.

[3] WHO-UNICEF, Policy statement on the use of vaccine vial monitors in immunization services. WHO/V/B/99, Vol. 18, WHO, Geneva, Switzerland, 1999.

[4] B. Y. Lee and L. A. Haidari, "The importance of vaccine supply chains to everyone in the vaccine world," Vaccine, vol. 35, no. 35 , pp. 4475-4479, 2017.

[5] S.-I. Chen, B. A. Norman, J. Rajgopal, and B. Y. Lee, "Passive cold devices for vaccine supply chains," Annals of Operations Research, vol. 230, no. 1, pp. 87-104, 2015.

[6] S. Lemmens, C. Decouttere, N. Vandaele, and M. Bernuzzi, “A review of integrated supply chain network design models: key issues for vaccine supply chains," Chemical Engineering Research and Design, vol. 109, pp. 366-384, 2016.

[7] E. Shittu, M. Harnly, S. Whitaker, and R. Miller, "Reorganizing Nigeria's vaccine supply chain reduces need for additional storage facilities, but more storage is required," Health Affairs, vol. 35, no. 2, pp. 293-300, 2016.

[8] A. J. Leidner, H. Fisun, S. Trimble, P. Lucas, C. Noblit, and J. M. Stevenson, "Evaluation of temperature stability among different types and grades of vaccine storage units: data from continuous temperature monitoring devices," Vaccine, vol. 38, no. 14, pp. 3008-3014, 2020.

[9] F. Lauton, A. Rothkopf, and R. Pibernik, "The value of entrant manufacturers: a study of competition and risk for donorfunded procurement of essential medicines," European Journal of Operational Research, vol. 272, no. 1, pp. 292-312, 2019.

[10] R. J. Tebbens, M. A. Pallansch, J. P. Alexander, and K. M Thompson, "Optimal vaccine stockpile design for an eradicated disease: application to polio," Vaccine, vol. 28, no. 26, pp. 4312-4327, 2010.

[11] M. Brison and Y. Letallec, "Transforming cold chain performance and management in lower-income countries," Vaccine, vol. 35, no. 17, pp. 2107-2109, 2017.

[12] Q. Lin, Q. Zhao, and B. Lev, "Cold chain transportation decision in the vaccine supply chain," European Journal of Operational Research, vol. 283, no. 1, pp. 182-195, 2020.

[13] T.L. Dai, S. H. Cho, and F. Q. Zhang, "Contracting for on-time delivery in the US influenza vaccine supply chain," SSRN Electronic Journal, vol. 18, no. 3, pp. 332-346, 2016.

[14] B.Z. Niu, Q. Y. Li, and L. Chen, "Exclusive vs. Competitive retailing: overseas vaccine supplier's channel selection considering profit and social responsibility objectives," Computers/Industrial Engineering, vol. 144, Article ID 106499, 2020.

[15] L. Xie and J. Ma, "Study the complexity and control of the recycling-supply chain of China's color TVs market based on the government subsidy," Communications in Nonlinear Science and Numerical Simulation, vol. 38, pp. 102-116, 2016.
[16] F. Wu and J. Ma, "The equilibrium, complexity analysis and control in epiphytic supply chain with product horizontal diversification," Nonlinear Dynamics, vol. 93, no. 4, pp. 2145-2158, 2018.

[17] Q.X. Li, X. L. Chen, and Y. M. Huang, "Complexity and entropy analysis of a multi-channel supply chain considering channel cooperation and service," Entropy, vol. 20, no. 12, p. 970, 2018.

[18] H. Tu, X. Mao, and X. Wang, "Complexity of a dynamic hybrid supply chain game model with a service factor," Nonlinear Dynamics, vol. 97, no. 4, pp. 2055-2066, 2019.

[19] J. Zhou and X. Chen, "The impact of service and channel integration on the stability and complexity of the supply chain," Complexity, vol. 2020, Article ID 8178947, 27 pages, 2020.

[20] J.C. Zou, K. U. Ranjit, A. Pratap, and Z. Z. Zhang, "Dynamics of a delayed SIR model for the transmission of PRRSV among a swine population," Advances in Difference Equations, vol. 2020, no. 1, pp. 1-30, 2020.

[21] Z. Zhang, J. Zou, R. K. Upadhyay, and A. Pratap, "Stability and Hopf bifurcation analysis of a delayed tobacco smoking model containing snuffing class," Advances in Difference Equations, vol. 2020, no. 1, 2020.

[22] M. Rajchakit and G. Rajchakit, "Mean square robust stability of stochastic switched discrete-time systems with convex polytopic uncertainties," Journal of Inequalities and Applications, vol. 2012, 2012.

[23] C. Li and S. Liu, "A robust optimization approach to reduce the bullwhip effect of supply chains with vendor order placement lead time delays in an uncertain environment," Applied Mathematical Modelling, vol. 37, no. 3, pp. 707-718, 2013.

[24] R. Saravanakumar, G. Rajchakit, C. K. Ahn, and H. R. Karimi, "Exponential stability, passivity, and dissipativity analysis of generalized neural networks with mixed time-varying delays," IEEE Transactions on Systems, Man, and Cybernetics: Systems, vol. 49, no. 2, pp. 395-405, 2019.

[25] P. Niamsup, M. Rajchakit, and G. Rajchakit, "Guaranteed cost control for switched recurrent neural networks with interval time-varying delay," Journal of Inequalities and Applications, vol. 2013, p. ID292, 2013.

[26] C. Maharajan, R. Raja, J. Cao, and G. Rajchakit, "Novel global robust exponential stability criterion for uncertain inertialtype BAM neural networks with discrete and distributed timevarying delays via Lagrange sense," Journal of the Franklin Institute, vol. 355, no. 11, pp. 4727-4754, 2018.

[27] P. Chanthorn, G. Rajchakit, J. Thipcha et al., "Robust stability of complex-valued stochastic neural networks with timevarying delays and parameter uncertainties," Mathematics, vol. 8 , no. 5 , p. $742,2020$.

[28] G. Rajchakit, A. Pratap, R. Raja, J. D. Cao, J. Alzabut, and C. X. Huang, "Hybrid control scheme for projective lag synchronization of riemann-liouville sense fractional order memristive BAM NeuralNetworks with mixed delays," Mathematics, vol. 7, no. 8, p. 759, 2019.

[29] A. Pratap, R. Raja, C. Sowmiya, O. Bagdasar, J. Cao, and G. Rajchakit, "Robust generalized Mittag-Leffler synchronization of fractional order neural networks with discontinuous activation and impulses," Neural Networks, vol. 103, pp. 128-141, 2018.

[30] T. Saravanakumar, V. J. Nirmala, R. Raja, J. Cao, and G. Lu, "Finite-time reliable dissipative control of neutral-type switched artificial neural networks with non-linear fault 
inputs and randomly occurring uncertainties," Asian Journal of Control, pp. 1-13, 2019.

[31] Q. Zhu, T. Saravanakumar, S. Gomathi, and S. M. Anthoni, "Finite-time extended dissipative based optimal guaranteed cost resilient control for switched neutral systems with stochastic actuator failures," IEEE Access, vol. 7, pp. 9028990303, 2019.

[32] Z.Z. Zhang and T. Zhao, "Bifurcation analysis of an e-SEIARS model with multiple delays for point-to-group worm propagation," Advances in Difference Equations, vol. 2019, 2019.

[33] Z. Zhang and Y. Wang, "Hopf bifurcation of a heroin model with time delay and saturated treatment function," Advances in Difference Equations, vol. 2019, no. 1, 2019.

[34] T. Saravanakumar, S. Marshal Anthoni, and Q. X. Zhu, "Resilient extended dissipative control for Markovian jump systems with partially known transition probabilities under actuator saturation," Journal of the Franklin Institute, vol. 357, no. 10, pp. 6197-6227, 2020.

[35] T. Saravanakumar, R. Sakthivel, P. Selvaraj, and S. M. Anthoni, "Dissipative analysis for discrete-time systems via fault-tolerant control against actuator failures," Complexity, vol. 21, no. S2, pp. 579-592, 2016.

[36] B. Sarkar, M. Omair, and N. Kim, “A cooperative advertising collaboration policy in supply chain management under uncertain conditions," Applied Soft Computing, vol. 88, pp. 1568-4946, 2020.

[37] Q.X. Li, X. L. Chen, and Y. M. Huang, "The stability and complexity analysis of a low-carbon supply chain considering fairness concern behavior and sales service," International Journal of Environmental Research $\backslash$ Public Health, vol. 16, no. 15, p. 2711, 2019.

[38] Q. Li, Y. Zhang, and Y. Huang, "The impacts of fairness concern and different business objectives on the complexity of dual-channel value chains," Complexity, vol. 2020, pp. 1-15, 2020. 